\title{
Helicobacter pylori: Beginning the second decade
}

\author{
ANN MATISKO BSC(HONS), ABR THOMSON MSC PHD FRCPC
}

\begin{abstract}
A MATISKO, ABR THOMSON. Helicobacter pylori: Beginning the second decade. Can J Gastroenterol 1995;9(6):333-343. 'Beginning the Second Decade' - a recent international meeting on Helicobacter pylori - was held in conjunction with the VIIth International Workshop on Gastroduodenal Pathology and H pylori and with the meeting of the European Helicobacter pylori Study Group in Houston, Texas from September 30 to October 1, 1994. A menu of 476 abstracts, published in the American Journal of Gastroenterology (1994;89:8), highlighted the explosion of advances in this area. The Houston meeting was followed by the Tenth World Congresses of Gastroenterology from October 2 to 7, 1994 in Los Angeles, California, again with scores of presentations and posters on topics ranging from the epidemiology of $\mathrm{H}$ pylori infection to steps towards the development of a human vaccine. All this was in addition to important new work presented earlier in 1994 in New Orleans during Digestive Diseases Week. In this digest of these important meetings, the authors will not regurgitate what the informed reader already knows, but will instead focus on the recent developments in important areas, providing selected key published references for background, and referring to this new work in abstract form which is at the cutting edge of "yesterday's tomorrow today".
\end{abstract}

Key Words: Duodenal ulcer, Eradication, Helicobacter pylori, Therapy

\section{Helicobacter pylori : le début de la deuxième décennie}

RÉSUMÉ : Le Début de la deuxième décennie (Beginning the Second Decade), cette récente réunion internationale sur Helicobacter pylori a eu lieu conjointement avec le septième atelier international sur la pathologie gastroduodénale et $H$. pylori et avec la réunion du Groupe d'étude européen sur Helicobacter pylori, à Houston au Texas, du 30 septembre au $1^{\text {er }}$ octobre 1994. Au menu, 476 résumés publiés dans l'American Journal of Gastroentorology (1994;89:8) qui a souligné les spectaculaires progrès accomplis dans ce domaine. La réunion de Houston a été suivie du $10^{\mathrm{e}}$ Congrès mondial de gastroentérologie qui avait lieu du 2 au 7 octobre 1994 à Los Angeles, en Californie, encore une fois accompagnée d'une foule de présentations et d'affiches sur des thèmes qui vari-

voir page suivante

An abbreviated list of references appears at the end of this paper. A full list is available from the corresponding author

Division of Gastroenterology, and Nutrition and Metabolism Research Group, University of Alberta, Edmonton, Alberta

Correspondence and reprints: Dr ABR Thomson, 519 Newton Research Building, University of Alberta, Edmonton, Alberta T6G 2C2. Telephone 403-492-6490, fax 403-492-7964, e-mail alan.thomson@ualberta.ca

Received for publication January 3, 1995. Accepted March 8, 1995
$\mathrm{T}$ HERE HAS BEEN AN EXPLOSION of research in the decade since Warren (1) and Marshall (2), in letters to the Lancet, drew attention to the association between Helicobacter pylori, a curved bacterium in the stomach, and gastritis. This area of research has advanced so rapidly that much of the important work has not yet been published, and by the 12 to 18 months that it takes to get to print, the information may be already superseded by further advances. Our apologies to workers in this field whose abstracts we have not mentioned, because we are subject to our own bias of bringing to the gastroenterologist what we believe to be the key new developments. Also, a number of conceptual aspects bordering on the philosophical will be addressed. These concepts include unresolved issues such as whether a follow-up endoscopy is needed to prove eradication of $\mathrm{H}$ pylori, why physicians have been so slow to become 'pylorites', and whether the National Institutes of Health (NIH) consensus to treat every $H$ pylori-positive duodenal ulcer (DU) patient on first encounter leaves any room for the many of us who slowly accepted the importance of maintenance therapy with $H$ pylori receptor antagonists only to have this concept like everything else in life in the '90s subjected to challenge and change.

We all grew up on the concept that ulcers were caused by acid (Schwartz's dictum that is three generations old) and could accept that defensive factors 
aient de l'épidémiologie de l'infection à H. pylori, à la préparation d'un vaccin. Tout ce matériel s'ajoutait aux importants travaux présentés plus tôt en 1994 à la Nouvelle-Orléans, durant la semaine des maladies digestives (Digestive Diseases Week). Dans leur synthèse de ces importantes réunions, les auteurs ne rabâcheront pas ce que le lecteur éclairé connaît déjà, mais s'attarderont plutôt sur les découvertes récentes dans les domaines de pointe, en fournissant les sources bibliographiques clés et en se référant aux nouveaux travaux, sous la forme de résumés de la plus brûlante actualité.

were likely to play some role in the pathogenesis of peptic disorders. How can an infection in the mucus in the stomach cause an ulcer in the duodenum? Wyatt and co-workers (3) showed that active duodenitis occurs in $H$ pylori-positive patients with gastric metaplasia. $H$ pylori infection in this aberrant gastric mucosa alters gastric physiology (4) and mucosal defence, leading to gastric or duodenal ulceration. H pylori infection is the major cause of chronic atrophic gastritis worldwide. Considering the long recognized association among chronic atrophic gastritis, intestinal metaplasia and gastric carcinoma, it should have been no surprise that an association was made between $H$ pylori and gastric cancer (GC) (5-7). Furthermore, H pylori-gastritis is not only the cause of primary B cell gastric lymphoma (mucosa-associated lymphoid tissue [MALToma]), but also treatment of $\mathrm{H}$ pylori causes the condition to regress or even to disappear (8). No wonder then that so much excitement has been raised at the possibility of curing peptic DU and gastric ulcers (GU). There is also the prospect of developing widescale vaccinations to prevent $H$ pylori infection and, by doing so, preventing some of the H pylori-related complications such as GC (9).

\section{EPIDEMIOLOGY}

Sensitive, specific and noninvasive $H$ pylori detection techniques have been applied to population studies to establish community prevalence of H pylori infection and to identify which populations are at risk. The route of transmission of $\mathrm{H}$ pylori remains controversial, but clearly $\mathrm{H}$ pylori is one of the most common chronic bacterial infections in humans worldwide (10).
The diagnostic tests for $\mathrm{H}$ pylori, and their strengths and limitations, will be considered in the 'Diagnosis' section. There is a widely held view that the so-called gold standard for $\mathrm{H}$ pylori diagnosis is demonstration of the organism on a suitably stained endoscopic gastric biopsy. Nonetheless, immunoglobulin (Ig) G serodiagnosis by commercially available immunoassays is useful for epidemiological studies. Noninvasive diagnosis may also be accomplished with ${ }^{14} \mathrm{C}$ or ${ }^{13} \mathrm{C}$ urea breath tests, or by relying upon the detection of $\mathrm{IgG}$ antibodies in saliva or urine. Exact prevalence data for $\mathrm{H}$ pylori are not yet available for Canada, but if we make the assumption that Canada remains 'developed' rather than 'developing', then the risk of $H$ pylori infection is likely about $1 \%$ per year of life (ie, $50 \%$ for a 50 -year-old gastroenterologist). Independent relationships also exist between $H$ pylori seropositivity and sharing a bed in childhood, housing density, locality of birth, adult social class and age (11).

The sources and transmission of $H$ pylori infection are unknown. H pylori has been isolated/observed only in the gastric mucus of humans, higher primates and possibly cats (12). Culturable forms of $H$ pylori can exist in water for $48 \mathrm{~h}$, suggesting that there may be environmental reservoirs $(13,14)$. Clustering of infection occurs within residential groups, including families $(15-17)$. There is evidence to support natural transmission by the oral-oral and fecal-oral routes, especially in populations with a high incidence of H pylori in childhood (18). H pylori has been cultured from the diarrheal stools of infected children and adults $(19,20)$ and from dental plaque in residents of India (21), but only rarely in the west $(22,23)$. In one study, $\mathrm{H}$ pylori was not transmitted from $\mathrm{H}$ pylori-infected mothers to their infants (24), which contradicts the oral transmission hypo-thesis.

\section{BACTERIAL PATHOGENIC FACTORS}

While a sizable proportion of our ever-ageing population becomes infected with $H$ pylori, only a portion of those develops complications such as DU, GU, GC or MALToma. This raises the likelihood that factors relating to the host, the organism or both influence the risk of an infected person developing ulceration or malignancy. The putative pathogenic considerations can be divided into colonization, persistence and disease-reducing factors (25).

Organism-related colonization factors include the production of urease, bacterial adhesins and bacterial receptors. An additional colonization factor is $H$ pylori motility; the two to six polar, sheathed filaments consist of two flagellum types: the cloned genes fla A and fla $\mathrm{B}$ (the former is essential for $H$ pylori motility) (26). H pylori urease is a nickel metalloenzyme associated with the bacterial surface. This enzyme facilitates colonization by producing a $\mathrm{pH}$ neutral microenvironment at the gastric surface in which $H$ pylori thrives. Urease expression may not be under the control of the nitrogen regulatory system (27).

Lewis blood group antigens are part of the blood group antigens determining blood group $\mathrm{O}$, and may mediate $\mathrm{H}$ pylori attachment to the gastric mucosa (28). Lewis B antigens are found in persons with blood group $\mathrm{O}$ (28). H pylori $63 \mathrm{kDa}$ exoenzyme S-like protein recognizes these lipids and enables the organism to attach to the gastric mucosa (29). No difference was found in the prevalence of $H$ pylori infection or peptic ulcers among patients expressing different Lewis phenotypes, but patients with the diffuse infiltrating type of carcinoma had higher frequency of the Lewis $(a-b+)$ phenotype (30).

Phosphatidylethanolamine and gangliotetraosylceramide are lipid re- 
ceptors for $\mathrm{H}$ pylori on the gastric mucosal membrane (31) and may enhance $H$ pylori colonization. There are many adhesins on the surface of $\mathrm{H}$ pylori, likely mediated by fucosylated structures in glycoproteins but not by sialylated ones (32). There may also be an external polysaccharide surface coat that has adherence properties (33). The surface-active phospholipid or surfactant coating the gastric mucosa may also coat $H$ pylori (34), and $H$ pylori may disrupt this layer (35).

An additional persistence factor for $H$ pylori infection is immune evasion, including the production of antibodies and protein-mediated suppression of the cellular immune response (36). There is conservation of a gene sequence between $H$ pylori and heat shock proteins (37), and thus there may be cross-reacting epitopes between bacterial and human proteins. Sialic acid residues on $H$ pylori are involved in the binding of $\mathrm{H}$ pylori to the epithelial surface of the stomach (38). Receptors for ligands have been found on neutrophils and on lymphocytes, and their expression is enhanced by inflammatory cytokines released during $H$ pylori infection (39).

The vacuolating cytotoxin of $\mathrm{H}$ pylori may also be an important diseaseinducing factor. Cytotoxin associated gene A (cagA) encodes a 128 kDa protein associated with cytotoxin production but does not directly mediate its activity; cagA has been cloned and sequenced $(40,41)$. This cagA protein is required for the expression of functional cytotoxin, and may be important for the folding and export of the $87 \mathrm{kDa}$ vacuolating cytotoxin gene (vacA) protein which mediates cytotoxin activity. There is no difference in the proportion of DU or GU subjects and $H$ pylori-positive asymptomatic volunteers for cagA $\operatorname{IgA}$ antibodies (42), which suggests that cagA is not essential for the development of DU $(41,43)$. Based on serological analysis, it had been suggested that only $\mathrm{H}$ pylori isolates expressing the cagA antigen are associated with DU. However, using polymerase chain reaction (PCR) methods on antral tissue, Labigne et al (43) showed that the cagA gene was found in $83 \%$ of DU patients and in $64 \%$ of non-DU patients. Indeed, none of the factors described above is definitely known to be pathogenic in the development of $\mathrm{H}$ pylori-associated disease; some strains may have greater virulence than others. For example, strains capable of activating chemiluminescence in neutrophils, that is, inducing an oxidative burst, are more prevalent in ulcer patients (44).

$H$ pylori may exert direct bacterial cytotoxicity on the foveolar surface epithelium, as well as indirect inflammatory cell-mediated damage on the proliferation zone of the gastric pits (45). The presence of $H$ pylori can induce cytoplasmic swelling, vacuolation, micropapillary changes, mucus depletion and erosion of the juxtaluminal cytoplasm, with overexpression of cathepsin $\mathrm{E}$ and human leukocyte antigen-DR (46). Expression of interleukin (IL)-8 in $H$ pylori-infected mucosa induces neutrophil chemotaxis (47). After eradication of H pylori, the degenerative lesions of the surface epithelium and the granulocytic activity of the gastritis regress (48-51). With the disappearance of neutrophils there is a concomitant decline in IgG plasma cells, but not in IgA plasma cells, lymphocytes or eosinophils $(48,49)$. With H pylori eradication the lymphoid infiltrates may (52) or may not (53) regress. Lymphoid follicles with germinal centres are common with $H$ pylori infection $(52,54)$. Gastric atrophy and intestinal metaplasia are also common, and the gastric metaplasia in the duodenal bulb persists after eradication of $H$ pylori $(48,55)$.

An additional reason that only some patients with $H$ pylori infection develop DU may relate to the bacterial load: the density of $H$ pylori as measured by quantitative culture is higher in DU, and cagA-positive strains colonized more densely than cagA-negative strains (56).

H pylori infection results in a humoral immune response, activates an oxidative burst in phagocytes, and may alter the function of mucosal $\mathrm{B}$ and $\mathrm{T}$ cells. An ELISA detecting a response to a recombinant fragment of the $128 \mathrm{kDa}$ protein (cagA) may prove to be useful to detect ulcerogenic $H$ pylori strains (57). Leukocyte adhesion and extravasation are increased with $H$ pylori infection as the result of the release of multiple factors (58) including a 150 $\mathrm{kDa}$ protein (59). An $\mathrm{H}$ pylori-associated $150 \mathrm{kDa}$ neutrophil activating protein has been identified that permits neutrophil adhesion to endothelial cells (59). The molecular mimicry of gastric antigens by $\mathrm{H}$ pylori may play a role in atrophic gastritis (60). Neutrophils then undergo an oxidative burst (44), possibly the result of the release of substance P (61), leading to mucosal cell injury.

DU patients given the free radical scavengers allopurinal and dimethyl sulphoxide have lower rates of ulcer recurrence compared with similar patients not given this treatment (62). $\mathrm{H}$ pylori induces natural killer cell activity (63) and stimulates peripheral blood lymphocytes (64). H pylori may stimulate the proliferation of mucosal $\mathrm{T}$ cells, followed by B cell proliferation (65). Mucosal IgA autoantibodies to IL-8 are produced in gastric organ cultures from $H$ pylori-infected patients (66), and these may represent a downregulatory response to the $H$ pylori-associated chronic infection (67). This gastric epithelial IL-8 gene expression may be associated with cagA but not vacA expression (68), and gamma-interferon secretion may also be important (69).

Thus, there is much speculation, but no proof, that any of these H pylori-related factors are responsible for the progression from $\mathrm{H}$ pylori colonization to $H$ pylori-associated diseases.

\section{GASTRIC PHYSIOLOGY}

Against the background of the previously accepted theory that peptic ulceration is somehow related to excess acid and pepsin, the possibility that ulcer disease might be due to an infection flies against all logic. If acid secretion is sufficiently inhibited for long enough, $100 \%$ of patients with DU will heal. How then are abnormalities in gastric physiology linked to peptic ulcer disease? There is now evidence that $H$ pylori infection may alter gas- 
tric physiology and may even enhance the potency of acid lowering proton pump inhibitors (PPIs) (70). One may consider the following points. The abnormalities in acid secretion appear to be related to increased levels of gastrin and reduced levels of somatostatin in tissue or blood (71). Compared with $\mathrm{H}$ pylori-negative, healthy, nonulcer volunteers, those who are $\mathrm{H}$ pylori-positive have a 20 to $30 \%$ higher mean basal serum gastrin concentration (72$75), 50$ to $100 \%$ increased mealstimulation gastrin concentrations $(72,73)$ and 200 to $300 \%$ higher gastrin concentrations in response to infusion with gastrin-releasing peptide (GRP) (75). H pylori-positive DU patients also have increased GRP-stimulated serum gastrin concentrations (75). Gastrin 34 is mainly duodenal in origin and is unaltered following $\mathrm{H}$ pylori eradication. Gastrin 17 rises after meals; this postprandial form of gastrin falls with $H$ pylori eradication (76). Eradication of $\mathrm{H}$ pylori normalizes the hypergastrinemia within two weeks of starting treatment for the infection $(72,76-82)$.

There is a reciprocal relationship between antral tissue $G$ and $D$ cells, and the release of gastrin from $G$ cells is under paracrine inhibitory control by somatostatin in $\mathrm{D}$ cells. In $\mathrm{H}$ pylori-infected volunteers or in DU patients, tissue levels of gastrin are increased, whereas somatostatin is decreased $(81,83)$. Surprisingly, the abnormal ratio of D cells:G cells in DU patients is not corrected when $\mathrm{H}$ pylori is treated (84).

How do these levels of serum or gastric tissue peptides relate to acid and pepsin secretion? H pylori-positive DU patients have higher basal and peak acid outputs than $H$ pylori-negative DU patients (73). But it is clear that in such a comparison patients need to be matched for age and for the presence of atrophic gastritis (74). In the same DU patient does acid secretion change with eradication of $\mathrm{H}$ pylori? Yes: initially elevated basal acid output falls dramatically $(4,8,75)$. In one report (8) neither the elevated maximal acid response to gastrin nor the accentuated parietal cell sensitivity to gastrin fell after $H$ pylori eradication. In another study, GRP-stimulated acid and gastrin responses were higher in $\mathrm{H}$ pylori-infected than in noninfected volunteers, and were even higher in $H$ pylori-infected DU patients (75). Within one month of eradicating $H$ pylori in healthy volunteers or DU patients the heightened gastrin, acid and pepsin responses to GRP fell or were normalized depending on the time the studies were done after eradication (75).

With $H$ pylori infection, then, there is an exaggerated release of gastrin and an exaggerated acid response to the extra gastrin (77). This suggests that there are $\mathrm{H}$ pylori-associated abnormalities in gastric $G$ or D cells, as well as in parietal cells. When acid is secreted, gastrin release is inhibited, and $H$ pylori infection may impair this normal inhibitory control of acid secretion $(72,86)$. On the one hand $H$ pylori does not appear to affect the maximal acid response to pentagastrin $(8,73,74,77)$. On the other hand $H$ pylori impairs the inhibitory control of gastric acid secretion $(72,77,86)$. It is reasonable to speculate that only $\mathrm{H}$ pylori-infected persons with an abnormal parietal cell mass will develop DU (71).

Other potentially important factors in gastric physiology that may relate to $H$ pylori infection include metaplasia, ascorbic acid and gastric motor activity. For $\mathrm{H}$ pylori to colonize the duodenum there must be gastric metaplasia in the duodenum, and this, in turn, may be due to an increased acid level in the duodenum $(55,87)$. Ascorbic acid is an antioxidant, and $H$ pylori infection is associated with a reduced concentration of ascorbic acid in gastric juice, which returns to normal upon eradication of H pylori $(88,89)$. H pylori infection may result in fewer phase III fronts of the migrating motor complex (90), but another group found that interdigestive and postprandial gastrointestinal mobility was unaffected by $\mathrm{H}$ pylori infection (91).

Thus, $H$ pylori infection results in increased basal acid secretion as well as increased secretion stimulated by GRP, and these changes may be due to an alteration in the ratio of $G$ cells:D cells in the gastric mucosa and to the presence of hypergastrinemia.

\section{DIAGNOSIS}

Noninvasive tests used to diagnose $H$ pylori generally have good sensitivity and specificity (92) compared with histological demonstration. The relative value of each test depends on the clinical objective for diagnosis (eg, epidemiological study versus symptomatic patient, or follow-up after attempted $H$ pylori eradication therapy). However, even histological demonstration of H pylori may be complicated by small false negative rates, particularly when moderate acid inhibition has been undertaken therapeutically, because the load of $\mathrm{H}$ pylori may be reduced and the distribution of $H$ pylori may be patchy or shift from the antrum to the gastric body. Whereas eradication therapy used to be defined as the loss of $\mathrm{H}$ pylori on biopsy or culture one month after therapy, there is a move to combine at least two different tests or multiple biopsies obtained at longer intervals (eg, two, three or six months, or longer) after completion of therapy. This may be necessary because there is a small rate of false negative testing, especially after an $H$ pylori eradication treatment course (93).

Rapid urease tests are simple and cost-effective for the diagnosis of $\mathrm{H} p \mathrm{p}$ lori on gastric biopsies, but the urease tests depend on an undefined $H$ pylori level, and sensitivity may fall from $100 \%$ pretreatment to $84 \%$ posttreatment for $\mathrm{H}$ pylori (94). About 16\% of persons who are actually infected with $H$ pylori may be falsely negative on rapid urease testing, yet may be rapidly diagnosed by phase contrast microscopy (95). Urea breath tests are useful even after attempted eradication therapy, but are limited by the availability of sophisticated instrumentation (96).

Most serological tests have used ELISAs on serum, and ELISAs have been developed for urine (97). New serological assays using a recombinant antigen or fragments of the $128 \mathrm{kDa}$ protein have also been developed (57). Serological testing may be used for long term monitoring of eradication therapy, but the serological tests need to be carefully validated, such as determining the change in titre that correctly reflects eradication (98). 
PCR may be useful to diagnose $H$ pylori $(99,100)$ with a high degree of sensitivity $(96 \%)$ and specificity (100\%) (101). PCR has been used to show $H$ pylori in dental plaques $(102,103)$, feces and gastric juice (104-109). PCR restriction fragment length polymorphism of urease or other genes may be used for ribotyping of $H$ pylori (110. 113). PCR has an advantage over $H$ pylori cultures because PCR results do not depend on transport conditions (106) and PCR results may be used to distinguish between reinfection versus recurrence (recrudescence) during relapse of H pylori infection (113).

\section{CLINICAL MANIFESTATIONS}

A quarter of asymptomatic $H$ pylori-infected persons have significant pathology on endoscopy (114), a proportion similar to those who are symptomatic. This is clearly an important issue because all $\mathrm{H}$ pylori-infected individuals may not require eradication therapy, and all dyspeptic patients may not suffer from DU or GU, even when infected with $\mathrm{H}$ pylori. Once virulence factors of $H$ pylori have been identified and confirmed in a clinical setting we will be in a better position to determine which $\mathrm{H}$ pylori-positive person requires further endoscopic study or $H$ pylori-eradication therapy. It remains unknown what factors determine the virulence of $H$ pylori in some patients.

The risk of developing GU and DU during chronic nonsteroidal antiinflammatory drug (NSAID) therapy is not influenced by $\mathrm{H}$ pylori infection (115), and NSAID therapy does not affect the rate of colonization with $\mathrm{H}$ pylori. The pathological changes in NSAID-associated gastritis differ from those observed in $\mathrm{H}$ pylori-associated gastritis, with more frequent foveolar hyperplasia, edema, telangiectasia of the lamina propria, fewer inflammatory cells and splaying of the muscularis mucosae in the former. Thus, $H$ pylori and NSAIDs are independent courses of distinctive mucosal damage, with neither influencing the damaging effect of the other (116).

Evidence for the association between gastric adenocarcinoma of the antrum and body (but not cardia) and
H pylori infection comes from epidemiological, serological and pathological studies (117-119). Eight studies in patients with early or advanced GC reported an increased prevalence of $\mathrm{H}$ pylori infection in diffuse (29 to 100\%), but especially in intestinal-type, cancer (47 to 89\%) (45). H pylori serology may be positive years before the development of GC (120). Infection with H pylori strains possessing $\operatorname{cag} \mathrm{A}$ is associated with increased risk of developing intestinal-type adenocarcinoma of the distal stomach among JapaneseAmerican men in Hawaii (121). It is particularly striking that all 205 patients with early diffuse GC studied by Fiocca et al (122) were $H$ pylori-infected.

In patients with high GC risk, there is a strong association between $H$ pylori infection and atrophy (relative risk $[R R]=15.0)$, and between intestinal metaplasia $(\mathrm{RR}=5.7)$ and dysplasia $(R R=4.0)(123)$. In fact, newer retrospective case-control studies confirm this association with $H$ pylori infection $(114,124,125)$ even after correction for the presence of atrophic gastritis (126). H pylori-associated gastritis may progress directly to the diffuse form of GC or may develop into intestinal-type cancer by first moving through a sequence of atrophy, intestinal metaplasia and dysplasia (45).

$H$ pylori infection and its associated inflammation result in the increased expression of proliferation antigens which are reduced with eradication (127). There is a higher prevalence of $H$ pylori strains that produce the 120 $\mathrm{kDa}$ cytotoxin in patients with GC (47); similarly, there is a higher prevalence of strains that produce neutralizing antibodies against the vacuolating cytotoxin. This consideration of hoststrain relationships is important because it will potentially help to identify which patient with which strain of $H$ pylori needs $H$ pylori eradication to prevent GC development. The mutagenic effect of H pylori may be the result of numerous processes, including the generation of reactive oxygen metabolites $(128,129)$ which are capable of damaging DNA, with the increased cell proliferation rates falling to normal after therapy (130).

$H$ pylori infection is also associated with the development of MALToma, probably through a multifactorial process including the development of lymphoid follicles in the gastric mucosa, possibly operating via T cells (65). $H$ pylori infection precedes the development of the gastric lymphoma (131). $\mathrm{H}$ pylori reduces the formation of lymphoid follicles (not present in normal mucosa) in the antrum and gastric body mucosa, and monoclonal lymphoid proliferation with characteristics of MALTomas shows histological regression and disappearance of monoclonality after $H$ pylori eradication (8). Of great importance is the finding that, in a double-blind randomized trial of 47 $H$ pylori-positive patients with MALToma, regression occurs in those treated for $H$ pylori but not in those given placebo (132).

\section{ERADICATION STRATEGIES}

Therapeutic regimens for eradication of $\mathrm{H}$ pylori include mono-, dual, triple or quadruple therapies. While clarithromycin alone results in an eradication rate (ER) of approximately $50 \%$, no monotherapy gives the $90 \%$ ER needed for clinical acceptance. While gastroenterologists may still be thinking of the peptic ulcer disease-H pylori association to focus on DU or GU, we predict that in the future our thinking will be that $H$ pylori is an infectious disease in which DU or GU is but one of several complications. Then, we will consider using one or two antibiotics to eradicate the infection, with an antisecretory agent to be used once to heal the ulcer - seldom to be used again because the ulcer will not recur unless the $H$ pylori infection recurs.

There has been a steady shift from the consensus to treat $H$ pylori-associated infection only in peptic ulcer patients with recurring disease (133), to the recent $\mathrm{NIH}$ recommendation to treat all DU patients for $H$ pylori at the time the ulcer is first diagnosed (134). In this review we will discuss what represents the optimal treatment regimens as of January 1995, and whom to treat 
(if not everyone with DU or GU and $H$ pylori infection).

Recent evidence continues to suggest that the best treatment regimens include omeprazole combined with an antibiotic such as amoxicillin, each given at least twice a day for two weeks, with or without a second antibiotic. Twice-daily dosing is more efficacious than once-daily regimens (135). An alternate useful regimen includes an $\mathrm{H}_{2}$-receptor antagonist combined with bismuth plus two antibiotics.

We begin with the background (136) that "eradication of $\mathrm{H}$ pylori reduces the rate of ulcer recurrence and rebleeding and minimizes both the direct and indirect cost, without the need for expensive maintenance therapy" (137). Eradication of $H$ pylori leaves the patient with about a $1 \%$ per year risk of reinfection with $H$ pylori and about a $2 \%$ risk of the ulcer recurring, versus at least a $50 \%$ chance that the DU or GU will recur over the next year if the $H$ pylori is not eradicated and if the patient is not on maintenance therapy with a potent acidlowering medication. Stress reduction and bland diets have gone by the wayside as part of the management of patients with DU or GU. However, smoking remains a concern because it may increase the risk of $H$ pylori infection, at least in African-American males living in New Orleans (138). Being male is also a risk factor for $\mathrm{H}$ pylori infection (139).

In the patient with DU or GU associated with $H$ pylori infection therapy, drugs such as $\mathrm{H}_{2}$-receptor antagonists or PPIs are given to heal the ulcer, while antibiotics (usually one or two) are also given (with or without bismuth) to eradicate $H$ pylori. Different investigators have used varying definitions of $H$ pylori eradication. The suggestion of defining eradication on the basis of one diagnostic method one month after stopping therapy has been extended to using two methods (140), and there is discussion that longer intervals of follow-up are needed before eradication can be accepted. Metronidazole resistance of $H$ pylori is well recognized, both before ('primary') and after ('secondary') therapy (141). Metronidazole resistance occurs in 7 to $49 \%$ of $\mathrm{H}$ pylori-infected patients (142), possibly due to a mutation in the H pylori's nitroreductase. Clarithromycin resistance is also recognized $(143,144)$. Primary amoxycillin resistance of $\mathrm{H}$ pylori is very rare (145).

Numerous regimens have been devised to eradicate $H$ pylori, some of which will be discussed briefly in the following sections.

Triple and quadruple therapy: Multiple reports of permeations and combinations of drugs to treat $H$ pylori have left most of us in a state of confusion. The recent meta-analysis of Mohamed and colleagues (146) has helped to clarify the use of various therapeutic approaches. Triple therapy including bismuth gives ERs of 75\% (bismuth + amoxicillin + metronidazole) and 87\% (bismuth + tetracycline + metronidazole). Triple therapy plus ranitidine gives higher rates of DU healing compared with ranitidine alone (147), supporting the NIH Consensus Statement that eradication of $\mathrm{H}$ pylori at the time of DU diagnosis represents appropriate therapy. Triple therapy (bismuth + tetracycline + imidazole) is continued for at least a week (148) but may be complicated by adverse affects in about a third of patients, poor compliance (the regimen comprises 18 pills a day) and the possible presence of metronidazole resistance.

Understandably ER is greatly influenced by compliance. Triple therapy combining bismuth with tetracycline gives highly variable ERs of $65 \%$ (bismuth + doxycycline + metronidazole) and $92 \%$ (bismuth + tetracycline + metronidazole). Reducing the dose of tetracycline does not appear to affect ER (ER of $92 \%$ with standard triple therapy of colloidal bismuth subcitrate [CBS] $108 \mathrm{mg}+$ tetracycline $500 \mathrm{mg}+$ metronidazole $250 \mathrm{mg}$ qid, versus ER of 96\% using CBS $108 \mathrm{mg}+$ tetracycline $250 \mathrm{mg}+$ metronidazole $250 \mathrm{mg}$ five times a day) (149). Again as expected, the ER of this triple therapy is affected by metronidazole resistance, with an ER of 85 to $98 \%$ with metronidazolesensitive $\mathrm{H}$ pylori using triple therapy of bismuth, tetracycline and metronidazole, versus an ER of 32 to $87 \%$ with metronidazole-resistant $H$ pylori (150). Other combinations of triple therapy using bismuth subsalicylate (BSS)/CBS, tetracycline and azithromycin, or furazolidone, amoxicillin and metronidazole give a lower ER of $58 \%$.

ERs for $H$ pylori are high with triple therapy. A study by Thjis and coworkers (151) showed that colloidal bismuth substrate $120 \mathrm{mg}$, tetracycline $250 \mathrm{mg}$ and metronidazole $250 \mathrm{mg}$ four times a day, for 15 days, had an ER of $93 \%$, and only $3 \%$ of patients with side effects stopped therapy. Other studies have given much lower ERs with triple therapy (148). An overall H pylori ER of $82 \%$ was noted for 67 studies including 78 treatment arms using triple therapy, but great variability was noted. The two-week ERs for bismuth plus amoxycillin plus a nitroimidazole was $75 \%$, and $86 \%$ for bismuth plus tetracycline plus a nitroimidazole. The addition of antisecretory agents to tetracycline triple therapy increases ERs to $91 \%$. ' $\mathrm{Tr}$ iple therapy' is a curious misnomer because a fourth acid lowering agent such as ranitidine needs to be given to heal the associated ulcer!

Much interest has recently been focused on a novel combination of ranitidine plus bismuth (RBC), used in conjunction with two antibiotics (152-156).

Dual or triple therapy with omeprazole is gaining wide acceptance. Omeprazole has mild topical anti-H pylori effects, due at least in part to omeprazole's action of inhibiting $H$ pylori ATPase, $H$ pylori histamine release and $H$ pylori urease activity. A PPI may also slow the degradation and thereby increase the local concentration of immunoglobulins, and enhance the $\mathrm{pH}$ environment for neutrophil function. Regimens including omeprazole give an ER of 58 to $85 \%$, depending on the total dose or dosing interval of omeprazole and the associated antibiotic, with an ER of 86 to $91 \%$ reported with omeprazole $20 \mathrm{mg}$ bid plus amoxicillin $1 \mathrm{~g}$ bid for 14 days (4).

Other combinations of PPI plus one or two antibiotics (known as 'PPI plus one' or 'PPI plus two') have been less successful when using omeprazole plus bismuth (ER 14\%), tetracycline (ER 
16\%), doxycycline (ER 0\%), erythromycin (ER 52\%), azithromycin (ER $62 \%$ ) or roxithromycin (ER 30\%). Triple therapy of PPI plus two antibiotics other than amoxicillin or clarithromycin has given widely variable ERs (26 to $75 \%$ ). Lansoprazole 'triples' reported in four trials (amoxicillin + tinidazole, or roxithromycin + metronidazole) give an ER of 77 to $82 \%$. If the combination of omeprazole and amoxicillin twice a day represents the current standard for $H$ pylori therapy, with an ER consistently over $80 \%$, triple combinations with clarithromycin $250 \mathrm{mg}$ bid plus omeprazole $20 \mathrm{mg}$ od or bid plus imidazole, amoxicillin, bismuth or tetracycline are attracting attention. New data on 'PPI plus two', including clarithromycin, are awaited with interest because of possible ERs of greater than $90 \%$. Compared with erythromycin, clarithromycin has improved bioavailability, a longer elimination half-life and fewer gastrointestinal adverse effects. The anti-H pylori activity of clarithromycin in vitro is very high, with the minimum inhibitory concentration required to inhibit $90 \%$ of H pylori (MIC 90) of less than $0.03 \mathrm{mg} /$ $\mathrm{mL}$ versus 0.03 for ampicillin, 4.00 for metronidazole, 25.0 for omeprazole, 12.5 for bismuth subcitrate or RBC, and 6.2 for lansoprazole. The MIC for many antibiotics, including clarithromycin, falls (and therefore anti-H pylori activity rises) with increasing $\mathrm{pH}$; for example, MIC for clarithromycin falls from $10.25 \mathrm{~g} / \mathrm{mL}$ at $\mathrm{pH} 5.5$ to 0.3 at $\mathrm{pH} 6.5$ (157). Pilot clinical trials of clarithromycin as monotherapy ( 1 to $2 \mathrm{~g} /$ day in divided doses) has given ERs of 15 to $54 \%(73,158)$. Because of the beneficial effect of alkaline $\mathrm{pH}$, which reduces the anti-H pylori effect of clarithromycin, the next logical step was to examine 'PPI plus one' using omeprazole and clarithromycin. Pilot clinical projects of omeprazole and clarithromycin gave an ER of 40 to $82 \%$ $(155,159)$. This was then followed by double-blind, randomized clinical trials using omeprazole up to $40 \mathrm{mg}$ daily and clarithromycin $500 \mathrm{mg}$ tid for 14 days in one study of 154 DU patients, which gave an ER of $83 \%$, and in a second study with 215 DU patients, which gave an ER of $74 \%$. The DU healing rates were high (95 to 99\%), and the 12 -month recurrence rates were low $(<12 \%)$.

Two small pilot trials reported ERs of 87 to $91 \%$ using omeprazole $20 \mathrm{mg}$ daily, clarithromycin $250 \mathrm{mg}$ tid and amoxicillin $2 \mathrm{~g} /$ day $(160,161)$. A multicentre, double-blind trial of omeprazole $20 \mathrm{mg}$ daily, amoxicillin $1 \mathrm{~g}$ bid and clarithromycin $500 \mathrm{mg}$ bid is in progress. Even lower doses such as clarithromycin $250 \mathrm{mg}$ bid, omeprazole up to $20 \mathrm{mg}$ bid and tinidazole $500 \mathrm{mg}$ bid have been reported to give an ER of 91 to $96 \%$ (161). In four studies, even one week of omeprazole, clarithromycin and tinidazole gives ER of 93 to $95 \%$ (162-164), raising the prospect of improving from an $80 \%$ ER with omeprazole and amoxicillin to $90 \%$ with omeprazole, clarithromycin and tinidazole.

RBC and clarithromycin $500 \mathrm{mg}$ tid for 14 days gave an ER of $82 \%$ in a large, double-blind study (153), while 'PPI plus two' using lansoprazole $30 \mathrm{mg}$ bid plus clarithromycin 400 to $1500 \mathrm{mg} /$ day gave a disappointing ER of 50 to $74 \%$ (165-167), and 'PPI plus two' with lansoprazole $30 \mathrm{mg}$ bid, clarithromycin $500 \mathrm{mg}$ bid plus amoxicillin $1 \mathrm{~g}$ bid for 14 days elicited a $96 \%$ ER in $25 \mathrm{H}$ pylori-positive DU/non-DU subjects (116).

Triple therapy with two antibiotics and omeprazole has provided good results: $91 \%$ eradication with omeprazole $40 \mathrm{mg}$ daily, amoxycillin $500 \mathrm{mg}$ tid plus metronidazole $400 \mathrm{mg}$ tid for 14 days, with a much lower ER of $47 \%$ in patients with metronidazole-resistant strains (168). ERs of $90 \%$ have also been reported for omeprazole $40 \mathrm{mg}$, amoxicillin $500 \mathrm{mg}$ tid plus clarithromycin $500 \mathrm{mg}$ tid (169).

Double therapy: An important metaanalysis of 264 studies reported between 1987 and 1993 showed that the combination of omeprazole $20 \mathrm{mg}$ bid plus amoxycillin for two weeks, or omeprazole at least $40 \mathrm{mg}$ daily plus amoxycillin for two weeks gave ERs of $83 \%$ and $85 \%$, respectively, while the combination of omeprazole, amoxicillin plus metronidazole gave an ER of 89\% (170). The overall $H$ pylori ER in studies of ulcer patients receiving omeprazole plus amoxycillin is $60 \%$ (148), but increasing the dose of omeprazole or giving it twice daily increases ERs. ERs of greater than $80 \%$ have been reported when omeprazole $40 \mathrm{mg}$ bid is given with amoxycillin $500 \mathrm{mg}$ bid for two weeks (171). A meta-analysis of treatment with omeprazole and amoxycillin showed that the most effective therapy is omeprazole $20 \mathrm{mg}$ bid plus amoxicillin $3 \mathrm{~g}$ daily for two weeks (135). Lower response rates are seen in older persons, smokers, noncompliant patients, and those with high acid outputs and previous exposure to omeprazole $(51,163,172)$. Advanced age, high scores of grade and of activity of gastritis, and GU disease are independent factors predicting treatment success with omeprazole plus amoxicillin (163). Omeprazole may have modest anti-H pylori effects on its own $(173,174)$, but its major effect is likely to improve antimicrobial action at an alkaline $\mathrm{pH}$ (175).

Lansoprazole has just been released in North America, and acceptable $H$ pylori ERs have been reported with this PPI when given with one or more antibiotics $(116,160,176,177)$.

$\mathrm{H}$ pylori eradication possibilities have been reviewed in detail (150). The degree of variability is great. The most constant ERs are achieved with bismuth/metronidazole tetracycline, or with bismuth, metronidazole and amoxycillin in triple therapy. When a twice-daily dose of omeprazole and amoxycillin is used, efficacy comparable with triple therapy is achieved with acceptable but still surprising variability between studies. In patients who are intolerant to amoxycillin, a suitable alternative is omeprazole $20 \mathrm{mg}$ bid plus clarithromycin $500 \mathrm{mg}$ tid for two weeks. According to the $1994 \mathrm{NIH}$ Consensus Statement, every patient with a DU or GU occurring in association with $H$ pylori infection should be considered for eradication therapy. The optimal monotherapy treatment regimen has not yet been found. Until then, dual therapy with omeprazole 40 $\mathrm{mg}$ bid plus amoxycillin $1 \mathrm{~g}$ bid or tid for two weeks is the treatment of choice for $H$ pylori infection in patients with DU or GU. Triple or quadruple regimens 
may be used for the approximately $15 \%$ of patients who do not eradicate $H$ pylori infection on PPI-antibiotic dual therapy. For those who fail to eradicate $H$ pylori infection after two trials of adequate drugs given for a least two weeks (taken by a compliant patient), maintenance therapy with antisecretory therapy needs to be offered. It is not clear whether the patient who has had $\mathrm{H}$ pylori-positive ulcer disease complicated by hemorrhage or perforation and who has had H pylori successfully eradicated can proceed with no antisecretory therapy, or whether maintenance antisecretory therapy remains prudent.

Reinfection or recrudescence and ulcer recurrence: When $H$ pylori infection recurs after previous successful eradication, there may have been reinfection with the same or different serospecies, or recrudescence of the original infection. The recrudescence may simply reflect insufficient sensitivity of the method or methods used to diagnose the presence of $\mathrm{H}$ pylori, which may have remained in smaller numbers in the gastric mucus or in some other protected reservoir. PCR $(178,179)$ or urea breath testing $(180,181)$ may help to detect small numbers of bacteria still present after attempted eradication therapy and may be used in future definitions of the success of eradication. The recurrence rate of $H$ pylori infection is approximately 0 to $2 \%$ per year $(182,183)$, although a higher reinfection rate may occur in developing countries $(184,185)$, possibly reflecting the higher annual acquisition rate. The recurrence rate will depend not only on the vigour with which the diagnosis was sought, but also how soon after eradication therapy the possibility of recurrence is examined. The current standard timing for eradication verification is one month post-treatment. The one-year H pylori recurrence rate in developing countries is $22 \%$, compared with $13 \%$ in so-called developed countries (148).

In the 27 DU studies quoted by Tytgat (150), the annual ulcer relapse rate was $58 \%$ (571 of 988) in H pylori-positive patients versus $2.6 \%$ (23 of 893 ) in $\mathrm{H}$ pylori-negative patients. Some of these apparently $H$ pylori-negative persons may have in fact been $\mathrm{H}$ pylori-positive; multiple or more sensitive methods such as PCR may have discerned this. GU annual relapse rates are also much lower in $\mathrm{H}$ pylori-negative $(\mathrm{O}$ to $7 \%$ ) compared with $H$ pylori-positive individuals (33 to $74 \%$ ). In patients with $H$ pylori-associated $\mathrm{GU}$, one-year recurrence rates were 48 to $51 \%$ without, versus 0 to $3 \%$ with, eradication therapy $(186,187)$.

Thus, there are excellent results to indicate that eradication therapy reduces the risk of ulcer recurrence. Does this translate to any reduction in ulcer complications? Yes: the risk of rebleeding is higher once the patient has initially presented with bleeding, and three studies have shown one-year follow-up of $0 \%$ rebleeding in patients treated with $\mathrm{H}$ pylori eradication compared with 22 to $37 \%$ who rebled when not given eradication therapy (188190). For unknown reasons, a lower than expected proportion of DU patients presenting with complications have proven $H$ pylori infection: $72 \%$ with bleeding DU $(191,192)$ and $48 \%$ with acute perforation $(192,193)$. It is essential to make a firm diagnosis; this is especially important in $\mathrm{H}$ pylori-negative patients with a bleeding DU because they may need to be maintained long term on an antisecretory drug.

Clearly longer follow-up is needed to determine whether $H$ pylori eradication patients presenting with a bleeding ulcer are free from the risk of recurrent bleeding over longer intervals, and whether they may be safe to be off antisecretory therapy completely. Certainly these data are encouraging, and it is difficult to think of a good reason not to use eradication therapy in this circumstance.

Nonresponse to attempted $\mathrm{H}$ pylori eradication: If the patient failed to eradicate $\mathrm{H}$ pylori with the first regimen of your choice, then a different treatment should be considered. For example, if a patient failed to respond to quadruple or triple therapy, omeprazole plus amoxicillin should be used; if omeprazole plus amoxicillin did not work, consider switching to omeprazole, amoxicillin plus clarithromycin, or to triple therapy. If the patient had been on omeprazole, which may interfere with $H$ pylori eradication (194), omeprazole should be stopped, replaced by an $\mathrm{H}_{2}$-receptor antagonist for two weeks, then treatment started with omeprazole plus amoxicillin, or omeprazole stopped and treatment with ranitidine plus triple therapy instituted.

Side effects and compliance: When triple therapy with bismuth and two antibiotics are used, side effects occur in about one-third of patients, with the most common complaints being nausea, and mouth and taste disturbances (148). Diarrhea occurs in about $10 \%$ of patients, and allergic skin reactions and candida infections occur in 1 to $2 \%$. Most side effects are mild and selflimiting, but in 3 to $7 \%$ of patients using triple therapy the adverse effects are sufficiently severe to stop treatment. In contrast, double therapy with omeprazole plus amoxycillin causes side effects (usually mild diarrhea) to occur in about $11 \%$ of patients, although $2 \%$ of patients will need to be withdrawn from treatment due to adverse events. The risk of developing pseudomembranous colitis from antibiotics used to eradicate $H$ pylori is unknown, but it must be exceedingly low. While it might be suspected that compliance is a problem with triple therapy because of the frequent adverse effects, such poor compliance has been difficult to prove.

\section{SPECIAL CONSIDERATIONS}

Nonulcer dyspepsia: There are reasonable points both for (195) and against (196) the treatment of $H$ pylori infection in dyspeptic patients who do not have a peptic ulcer, that is, the patient with 'functional' or nonulcer dyspepsia (NUD). When a patient with ulcer-like symptoms is endoscoped sometimes an ulcer is found. If there is an ulcer it is treated and we expect the symptoms to go away. If there is no ulcer, but there is $\mathrm{H}$ pylori-associated chronic active antral gastritis, does this mean that the patient also has an ulcer diathesis, that an ulcer will form at a later date and that we just picked the wrong time to endoscope? We recognize that some patients 
with gastritis have no symptoms, just like some patients with an ulcer have no symptoms. And we really don't know what causes pain in a patient with an ulcer. So with all this overlap between symptomatic versus asymptomatic presentations of $\mathrm{H}$ pylori-infected patients, is it any surprise that there is controversy? Until ulcer-specific serotypes of $H$ pylori are found we are left with doing the best we can, interpreting the available literature. Some NUD patients are symptom-free at one year after eradication therapy (197); some NUD patients in whom pain predominates improve (149). Therefore, in the patient with ulcer-like symptoms and $H$ pylori-associated antral gastritis for which no other cause for dyspepsia can be found (such as reflux esophagitis or cholelithiasis), it seems reasonable to advise the patient of the controversy, the options of no treatment versus trials of promotility or antisecretory agents and the possibility of considering $\mathrm{H}$ pylori eradication therapy. Certainly this is a view shared by many of our colleagues (198).

Because acid secretion falls when H pylori infection is treated, some physician panelists are even suggesting that $H$ pylori infection should be treated in a patient with gastroesophageal reflux disease. This will remain as controversial an issue, obviously, as treating the NUD patient or the asymptomatic person who is concerned about being H pylori positive by serology. Clearly more work is needed to identify which subgroup of nonulcer $H$ pylori-infected individuals should be offered $H$ pylori eradication therapy.

Risk of gastroduodenal disease in $H$ pylori-positive asymptomatic persons: Totally asymptomatic British blood donors positive for $\mathrm{H}$ pylori by serology were asked to have an endoscopy, and $24 \%$ had DU or GU, $47 \%$ had antral gastritis or erosions, and 1\% had GC (114). Similar findings, including GC, were reported for San Marino (199), and an even higher rate of endoscopic abnormalities (80\%) was reported for Dublin, Ireland (200). With such a high rate of pathology in asymptomatic persons who are positive for $\mathrm{H}$ pylori is it any wonder that $\mathrm{H}$ py- lori screening may be studied for the possible early detection of DU or GU, and even GC in a low incidence country like Canada? And is it any surprise that we may consider $H$ pylori screening in the future for all dyspeptic patients, performing endoscopy only on those who are positive?

\section{VACCINATION}

$\mathrm{H}$ pylori urease and other recently cloned antigens may serve as useful antigens for vaccination, and much attention is focused on vaccine adjuvants and delivery systems (201). Oral immunization against $H$ pylori has been possible in a mouse model of $H$ pylori $(202,203)$, and oral immunization of mice with $H$ pylori urease or with urease subunits are protective against Helicobacter felis infection $(204,205)$. Nontoxic adjuvants have been developed (206), and vaccination of mice may actually clear the $\mathrm{H}$ felis infection (9,204,205). These developments point towards the possibility of using immunization as treatment as well as for prevention of $\mathrm{H}$ pylori.

\section{A PERSONAL PERSPECTIVE OF YESTERDAY'S TOMORROW TODAY}

When keypad voting was used at two Canadian Association of Gastroenterology-sponsored continued medical education meetings of Canadian gastroenterologists in 1994, over $80 \%$ of attending physicians indicated that they would treat at least some $H$ pylori-positive patients with eradication therapy. Now that simpler and more convenient regimens have been developed there can be less concern about side effects or patient compliance and acceptance. Cost-benefit considerations in the Canadian context have confirmed the major final saving of using eradication therapy in patients with DU disease (personal communication). Furthermore, the one-time cost of eradication therapy is little more than the cost of a single healing course of antisecretory therapy. In addition, $H$ pylori eradication therapy eliminates the need for maintenance therapy with antisecretory agents, except in the small minority of patients who fail to eradicate after one or two courses of therapy.

While guidelines have been developed for indications for $H$ pylori eradication therapy, such as "recurrent $H$ pylori-positive peptic ulcer disease in younger patients without risks for complications; peptic ulcer refractory to antisecretory drugs; and failed prophylactic therapy" (148), the members of the NIH Consensus Development Conference held February 7 to 9, 1994 suggested "...that all H pylori-infected patients with GU or DU should receive antihelicobacter therapy in addition to traditional anti-ulcer medications as primary therapy for ulcer disease, whether at initial presentation or at the time of recurrence" (207).

The slowness of some physicians to adopt $H$ pylori eradication therapy may be due to their having accepted Schwartz's 84-year-old dictum, 'no acid, no ulcer'; they may find it difficult to accept that this is incorrect and that $H$ pylori explains peptic ulcer disease. Perhaps those who are hesitant to shift to a new paradigm for the ' 90 s would be prepared to accept 'no acid, no H pylori, no ulcer'! With the important work of McColl and Deltenre (71) and others linking H pylori to abnormalities in gastric physiology - basal and peak acid output, hypergastrinemia and hypersensitivity of the parietal cell - the 'pylorites' have joined the 'acidologists', and an accepted spectrum of pathophysiological abnormalities increasingly is becoming accepted. Modern Canadian gastroenterologists reject phenomenology and base most of their therapeutic decisions on scientific facts. As these facts develop and are rapidly communicated, our allowed reaction time becomes less and less, and we need to move quickly to evaluate new findings and to bring these developments to benefit the patients for whom we care.

ACKNOWLEDGEMENTS: The authors thank Marlene Hoffmann for her excellent secretarial and editing assistance.

\section{REFERENCES}

1. Lancet 1983;i:1273. (Lett) 
2. Lancet $1983 ; 1: 1273-5$. (Lett)

3. J Clin Pathol 1987;40:841-8.

4. Gut 1993;34:1060-5.

5. N Engl J Med 1991;325:1132-6.

6. N Engl J Med 1991;325:1127-31.

7. BMJ 1991;302:1302-5.

8. Lancet 1991;ii:1175-6.

9. Lancet 1994;i:914-5.

10. Curr Opin Gastroenterol 1994;10(Suppl 1):6-11.

11. Epidemiol Infect 1993;111:63-70.

12. Gastroenterology 1994;106:89A. (Abst)

13. Appl Environ Microbiol 1993;59:1231-5.

14. Lancet 1991;337:1503-6.

15. Gut 1993;34:1348-50.

16. J Infect Dis 1993;168:1544-8.

17. Gut 1994:35:313-6.

18. Abstract 83. The VIIth Workshop on Gastroduodenal Pathology and Helicobacter pylori, Houston, Texas, September-October 1994.

19. Lancet 1992;340:1194-5.

20. Gastroenterology 1994;107:1671-4.

21. Scand J Gastroenterol 1991;26:1205-8.

22. J Clin Microbiol 1989;27:1397-8.

23. Eur J Clin Microbiol Infect Dis 1993;12:282-5.

24. Am J Gastroenterol 1994;89:1334. (Abst 198)

25. Curr Opin Gastroenterol 1994;10(Suppl 1):17-21.

26. J Bacteriol 1993;175:3278-88.

27. Infect Immunol 1993;61:2563-9.

28. Science 1993;262:1892-5.

29. Infect Immun 1993;61:2474-8.

30. Am J Gastroenterol 1994;89:1331. (Abst A183)

31. Infect Immun 1993;61:2632-8.

32. Proc Natl Acad Sci USA 1993;90:2035-9.

33. Infect Immun 1993;61:2732-6.

34. Gut 1993;34:588-93.

35. Gastroenterology 1993;105:1698-704.

36. Med Microbiol Immunol (Berl) 1993;182:63-76.

37. Mol Microbiol 1993;9:645-52.

38. Am J Gastric 1994;89:1325.

39. Am J Gastric 1994;89:1337.

40. Infect Immun 1993;61:1799-809.

41. Proc Natl Acad Sci USA 1993;90:5791-5.

42. Abstract 151. The VIIth Workshop on Gastroduodenal Pathology and Helicobacter pylori, Houston, Texas, September-October 1994.

43. Am J Gastric 1994;89:1326.

44. Gut 1993;34:599-603.

45. Curr Opin Gastroenterol 1994;10(Suppl 1):35-40.

46. Scand J Gastroenterol 1994;205:11-21.

47. J Clin Pathol 1994;47:61-6.

48. Scand J Gastroenterol 1994;29 (Suppl 201):28-34.

49. Mod Pathol 1993;6:281-9.

50. Eur J Gastroenterol Hepatol 1993;5:701-5.
51. Eur J Gastroenterol Hepatol 1993;5:325-31.

52. Abstract 260. The VIIth Workshop on Gastroduodenal Pathology and Helicobacter pylori, Houston, Texas, September-October 1994.

53. Abstract 249. The VIIth Workshop on Gastroduodenal Pathology and Helicobacter pylori, Houston, Texas, September-October 1994.

54. Hum Pathol 1993;24:577-83.

55. Gut 1993;34:1510-4.

56. Am J Gastric 1994;89:1322.

57. Eur J Clin Microbiol Infect Dis 1993;12:739-45.

58. Gastroenterology 1993;105:1431-40.

59. Am J Gastric 1994;89:1338.

60. Am J Gastric 1994;89:1329.

61. Abstract 229. The VIIth Workshop on Gastroduodenal Pathology and Helicobacter pylori, Houston, Texas, September-October 1994.

62. Intern Med 1993;32:359-64.

63. Infect Immun 1993;61:3012-6.

64. Int J Med Microbiol Virol Parasitol Infect Dis 1993;280:166-76.

65. Lancet 1993;342:571-4.

66. Scand J Immunol 1993;37:65-70.

67. Curr Opin Gastroenterol 1994;10(Suppl 1):26-9.

68. Abstract 207. The VIIth Workshop on Gastroduodenal Pathology and Helicobacter pylori, Houston, Texas, September-October 1994.

69. Abstract 220. The VIIth Workshop on Gastroduodenal Pathology and Helicobacter pylori, Houston, Texas, September-October 1994.

70. Scand J Gastroenterol 1994;12 (Suppl 29):1065-9.

71. Curr Opin Gastroenterol 1994;10(Suppl 1):22-5.

72. Dig Dis Sci 1993;38:1681-7.

73. Am J Gastroenterol 1993;88:2038-43.

74. Gut 1993;34:1032-7.

75. Gut 1993;34(Suppl 4):S49.

76. Gut 1993;34:757-61.

77. Gut 1994;35(Suppl 2):S4. (Abst)

78. Eur J Gastroenterol Hepatol 1993;5:13-6.

79. Scand J Gastroenterol 1993;28:690-4.

80. Gut 1993;34:888-92.

81. Scand J Gastroenterol 1993;28:858-64.

82. Am J Gastroenterol 1993;88:440-2.

83. Gut 1994;35:615-8.

84. Gastroenterology 1993;104:1655-60.

85. Gut 1993;34:888-92.

86. Scand J Gastroenterol 1993;28:999-1004.

87. Eur J Gastroenterol Hepatol 1993;5:1-8.

88. Gut 1994;35:317-23.

89. Gut 1993;34:1038-41.

90. Dig Dis Sci 1993;38:2255-61.

91. Am J Gastroenterol 1993;88:364-74.

92. Am J Gastroenterol 1994;89:S116-28.

93. Am J Gastroenterol 1994;89:1365. (Abst 322)
94. Braz J Med Biol Res 1993;26:699-702.

95. Am J Gastric 1994;89:1350.

96. Goodwin CS, Worsley BW, eds. Helicobacter pylori: Biology and Clinical Practice. Boca Raton: CRC Press, 1993:307-27.

97. J Clin Microbiol 1993;31:2174-7.

98. Goodwin CS, Worsley, BW, eds. Helicobacter pylori: Biology and Clinical Practice. Boca Raton: CRC Press, 1993:285-305.

99. Eur J Clin Microbiol Infect Dis 1993;12:367-71.

100. J Clin Microbiol 1993;31:1918-20.

101. Clin Infect Dis 1993;16:367-71.

102. J Clin Microbiol 1993;31:783-7.

103. Abstract 54. The VIIth Workshop on Gastroduodenal Pathology and Helicobacter pylori, Houston, Texas, September-October 1994.

104. Abstract 35. The VIIth Workshop on Gastroduodenal Pathology and Helicobacter pylori, Houston, Texas, September-October 1994.

105. Abstract 35. The VIIth Workshop on Gastroduodenal Pathology and Helicobacter pylori, Houston, Texas, September-October 1994.

106. Abstract 40. The VIIth Workshop on Gastroduodenal Pathology and Helicobacter pylori, Houston, Texas, September-October 1994.

107. Abstract 48. The VIIth Workshop on Gastroduodenal Pathology and Helicobacter pylori, Houston, Texas, September-October 1994.

108. Abstract 52. The VIIth Workshop on Gastroduodenal Pathology and Helicobacter pylori, Houston, Texas, September-October 1994.

109. Lancet 1993;i:447. (Lett)

110. J Clin Microbiol 1993;31:1420-5.

111. Abstract 43. The VIIth Workshop on Gastroduodenal Pathology and Helicobacter pylori, Houston, Texas, September-October 1994.

112. Abstract 29. The VIIth Workshop on Gastroduodenal Pathology and Helicobacter pylori, Houston, Texas, September-October 1994.

113. Abstract 42. The VIIth Workshop on Gastroduodenal Pathology and Helicobacter pylori, Houston, Texas, September-October 1994.

114. Am J Gastroenterol 1994;89:1314. (Abst 118)

115. Am J Gastroenterol 1994;89:203-7.

116. Abstract 327. The VIIth Workshop on Gastroduodenal Pathology and Helicobacter pylori, Houston, Texas, September-October 1994.

117. Gastroenterology 1993;105:1098-103.

118. Lancet 1993;341:1359-62.

119. Eur J Gastroenterol Hepatol 1993;5(Suppl 2):S2-8.

120. Abstract 292. The VIIth Workshop on Gastroduodenal Pathology and 
Helicobacter pylori, Houston, Texas, September-October 1994.

121. Abstract 283. The VIIth Workshop on Gastroduodenal Pathology and Helicobacter pylori, Houston, Texas, September-October 1994.

122. Am J Gastroenterol 1994;89:1357. (Abst 290)

123. Cancer 1993;71:297-301.

124. Abstract 286. The VIIth Workshop on Gastroduodenal Pathology and Helicobacter pylori, Houston, Texas, September-October 1994.

125. Abstract 438. The VIIth Workshop on Gastroduodenal Pathology and Helicobacter pylori, Houston, Texas, September-October 1994.

126. Abstract 453. The VIIth Workshop on Gastroduodenal Pathology and Helicobacter pylori, Houston, Texas, September-October 1994.

127. Am J Gastroenterol 1993;88:1870-5.

128. Gut 1993;35:179-85.

129. Abstract 288. The VIIth Workshop on Gastroduodenal Pathology and Helicobacter pylori, Houston, Texas, September-October 1994.

130. Abstract 298. The VIIth Workshop on Gastroduodenal Pathology and Helicobacter pylori, Houston, Texas, September-October 1994.

131. N Engl J Med 1994;330:1267-71.

132. Abstract 282. The VIIth Workshop on Gastroduodenal Pathology and Helicobacter pylori, Houston, Texas, September-October 1994.

133. Working Party Report World Congress of Gastroenterology. Oxford: Blackwell Scientific Publications, 1990:36-47.

134. JAMA 1994;272:65-9.

135. Abstract 395. The VIIth Workshop on Gastroduodenal Pathology and Helicobacter pylori, Houston, Texas, September-October 1994.

136. Postgrad Med J 1992;68:549-57.

137. Curr Opin Gastroenterol 1994;10(Suppl 1):46-52.

138. Am J Gastric 1994;89:1303.

139. Am J Gastric 1994;89:1312.

140. Eur J Gastroenterol Hepatol 1993;5:963-7.

141. Ir J Med Sci 1993;162:91-4.

142. Eur J Clin Microbiol Infect Dis 1992;11:777-81.

143. Abstract 390. The VIIth Workshop on Gastroduodenal Pathology and Helicobacter pylori, Houston, Texas, September-October 1994.

144. Abstract 417. The VIIth Workshop on Gastroduodenal Pathology and Helicobacter pylori, Houston, Texas, September-October 1994.
145. Rev Esp Enferm Dig 1990;78:P219. (Abst)

146. Eradication of Helicobacter pylori (HP): A Meta-Analysis. Presented at the 10th World Congresses of Gastroenterology, Los Angeles, October 1994.

147. Ann Intern Med 1991;115:266-9.

148. Aliment Pharmacol Ther 1994;8:369-89.

149. Abstract 320. The VIIth Workshop on Gastroduodenal Pathology and Helicobacter pylori, Houston, Texas, September-October 1994.

150. Aliment Pharmacol Ther 1994;8:359-68.

151. Scand J Gastroenterol 1993;28:934-8.

152. Abstract 386. The VIIth Workshop on Gastroduodenal Pathology and Helicobacter pylori, Houston, Texas, September-October 1994.

153. Am J Gastric 1994;89:1390.

154. Am J Gastroenterol 1994;89:1399. (Abst 456)

155. Am J Gastroenterol 1994;89:1382. (Abst 387)

156. Abstract 388. The VIIth Workshop on Gastroduodenal Pathology and Helicobacter pylori, Houston, Texas, September-October 1994.

157. Gut 1995;37(Suppl 1):361. (Abst 91)

158. J Clin Gastroenterol 1994;16:292-4.

159. Aliment Pharmacol Ther 1993; 7:149-53

160. Clin Ther 1993;15(Suppl B):32-6.

161. Gut 1994;35(Suppl 2):562 .

162. Eur J Gastroenterol 1994;6:773-7.

163. Am J Gastric 1994;89:1376.

164. Am J Gastroenterol 1994;89:1400. (Abst 461)

165. Am J Gastroenterol 1994;89:1388. (Abst 411)

166. Am J Gastroenterol 1994;89:1389. (Abst 415)

167. Am J Gastroenterol 1994;89:1373. (Abst 354)

168. Q J Med 1993;86:743-50.

169. Gut 1994;35:15-8.

170. Am J Gastroenterol 1994;87:1716-27.

171. Am J Gastroenterol 1993;88:491-5.

172. Abstract 367. The VIIth Workshop on Gastroduodenal Pathology and Helicobacter pylori, Houston, Texas, September-October 1994.

173. Antimicrob Agents Chemother 1991;35:490-6.

174. Eur J Gastroenterol Hepatol 1993;5:683-5.

175. Scand J Infect Dis 1989;21:219-23.

176. Abstract 346. The VIIth Workshop on Gastroduodenal Pathology and Helicobacter pylori, Houston, Texas, September-October 1994.
177. Abstract 428. The VIIth Workshop on Gastroduodenal Pathology and Helicobacter pylori, Houston, Texas, September-October 1994.

178. Abstract 410. The VIIth Workshop on Gastroduodenal Pathology and Helicobacter pylori, Houston, Texas, September-October 1994.

179. Abstract 411. The VIIth Workshop on Gastroduodenal Pathology and Helicobacter pylori, Houston, Texas, September-October 1994.

180. Abstract 361. The VIIth Workshop on Gastroduodenal Pathology and Helicobacter pylori, Houston, Texas, September-October 1994.

181. Abstract 308. The VIIth Workshop on Gastroduodenal Pathology and Helicobacter pylori, Houston, Texas, September-October 1994.

182. Abstract 363. The VIIth Workshop on Gastroduodenal Pathology and Helicobacter pylori, Houston, Texas, September-October 1994.

183. Am J Gastric 1994;89:1365.

184. Scand J Gastroenterol 1992;27:362-6.

185. Abstract 329. The VIIth Workshop on Gastroduodenal Pathology and Helicobacter pylori, Houston, Texas, September-October 1994.

186. Gastroenterology 1993;104:A40. (Abst)

187. Scand J Gastroenterol 1993;28 (Suppl 197):70.

188. Scand J Gastroenterol 1993;28:939-42.

189. Proceedings of the 11 United European Gastroenterology Week, Barcelona, 1993:A96. (Abst)

190. Proceedings of the 11 United European Gastroenterology Week, Barcelona, 1993:A3. (Abst)

191. Gastroenterology 1992;102:A90. (Abst)

192. Gastroenterology 1993;104:A64. (Abst)

193. Gut 1992;33(Suppl 1):S67.

194. Am J Gastroenterol 1994;89:1376. (Abst 366)

195. Gastroenterol Clin North Am 1993;22:141-51.

196. Gastroenterol Clin North Am 1993;22:153-67.

197. Gastroenterology 1992;103:341. (Lett)

198. Abstract 454. The VIIth Workshop on Gastroduodenal Pathology and Helicobacter pylori, Houston, Texas, September-October 1994.

199. Am J Gastric 1994;89:1307.

200. Am J Gastric 1994;89:1302.

201. Curr Opin Gastroenterol 1994;10(Suppl 1):53-7.

202. Vaccine 1993;11:637-42. 
203. Int J Med Microbiol Virol Parasitol Infect Dis 1993;280:155-65.

204. Gastroenterology 1994;107:1002-4.

205. Abstract 231. The VIIth Workshop on
Gastroduodenal Pathology and

Helicobacter pylori, Houston, Texas,

September-October 1994.

206. Abstract 203. The VIIth Workshop on
Gastroduodenal Pathology and

Helicobacter pylori, Houston, Texas, September-October 1994.

207. Am J Gastroenterol 1994;89:1137-9. (Edit) 


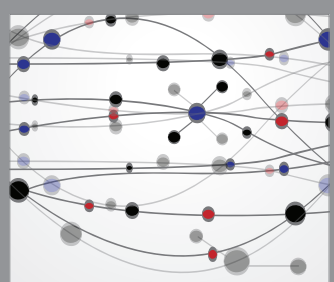

The Scientific World Journal
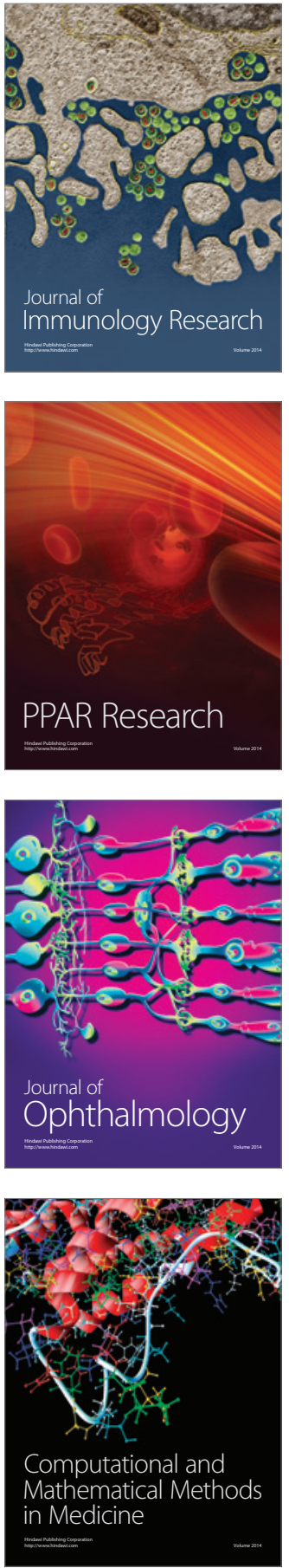

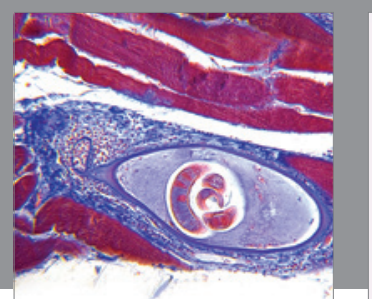

Gastroenterology Research and Practice

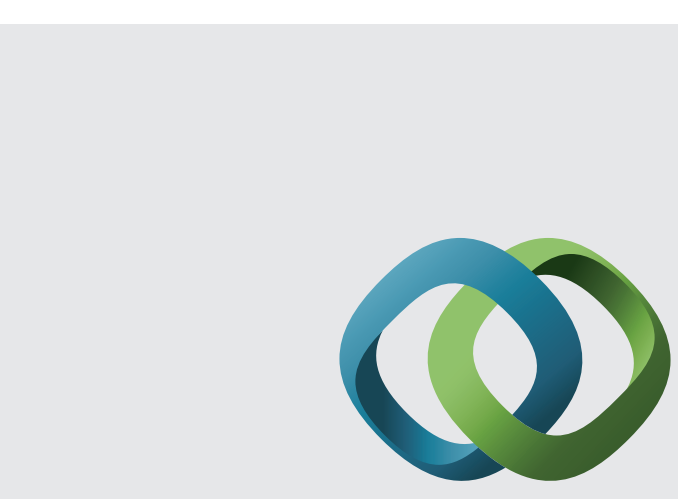

\section{Hindawi}

Submit your manuscripts at

http://www.hindawi.com
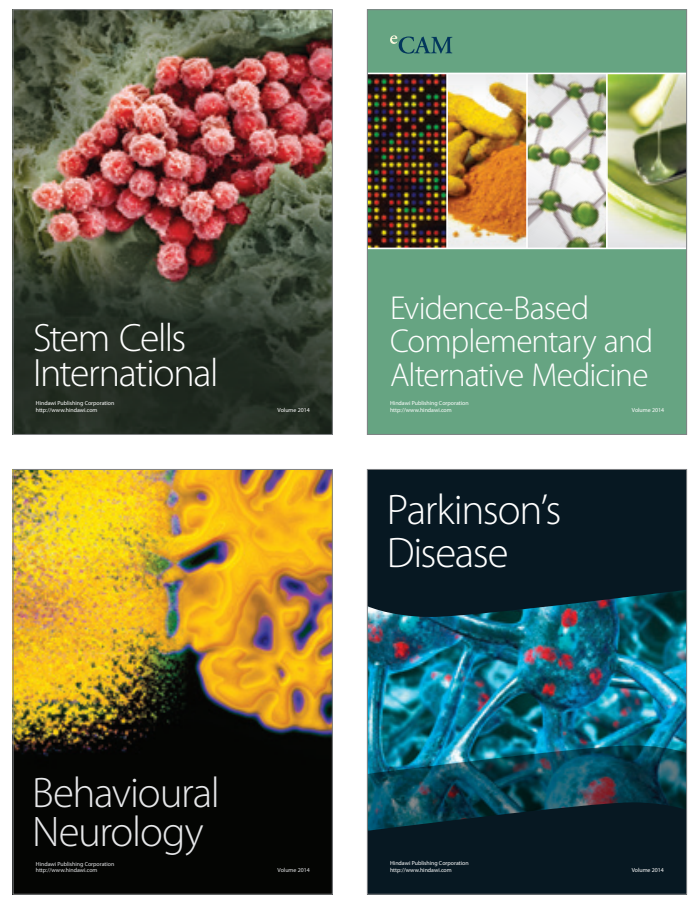
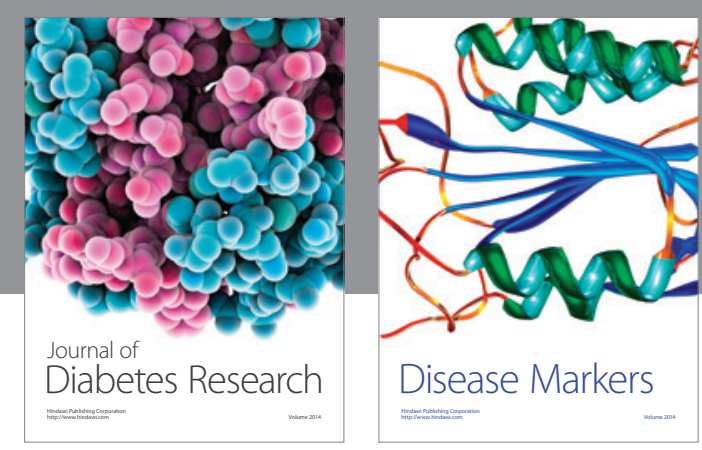

Disease Markers
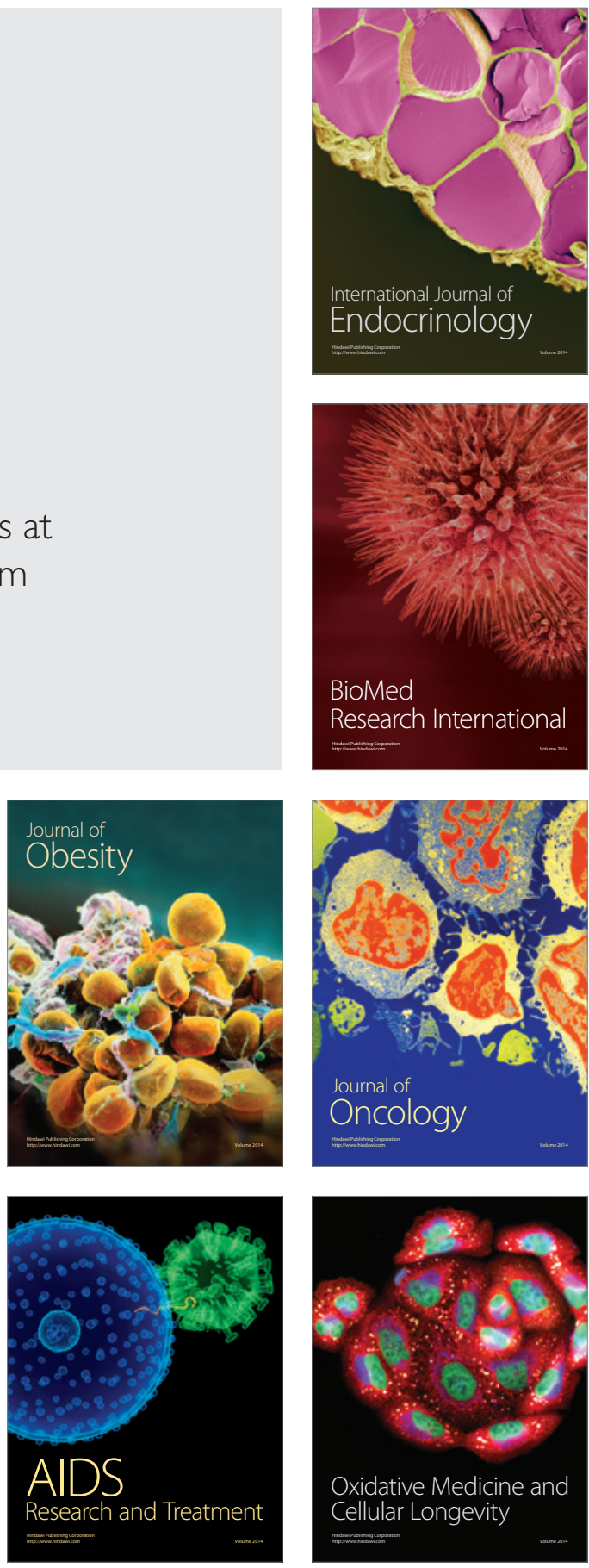